\title{
Simulating the recent star formation history in the halo of NGC $5128^{\star}$
}

\author{
M. Rejkuba ${ }^{1}$, L. Greggio ${ }^{2}$, and M. Zoccali ${ }^{1,3}$ \\ 1 European Southern Observatory, Karl-Schwarzschild-Strasse 2, 85748 Garching, Germany \\ e-mail: mzoccali@eso.org \\ 2 INAF, Osservatorio Astronomico di Padova, Vicolo dell'Osservatorio 5, 35122 Padova, Italy \\ e-mail: greggio@pd.astro.it \\ 3 Departamento de Astronomía y Astrofísica, Pontificia Universidad Católica de Chile, Casilla 306, Santiago 22, Chile \\ and Princeton University Observatory, Peyton Hall, Princeton, NJ 08544-1001, USA
}

Received 15 August 2003 / Accepted 6 November 2003

\begin{abstract}
Simulated color-magnitude diagrams are used to investigate the recent star formation history in NGC 5128. The comparison of the simulations with the observed $U V$ color-magnitude diagram for a field in the north-eastern shell, where recent star formation is present, constrains the initial mass function (IMF) and the duration of the star formation episode. The star formation burst is still on-going or at most has stopped some 2 Myr ago. The look-back time on the main sequence is set by the incompleteness of the $U$-band observations at $V \sim 26$, and is of the order of $50 \mathrm{Myr}$. The post main sequence phases have a look-back time up to $100 \mathrm{Myr}$, setting the maximum observable time for the star formation in this field. The comparison of the observed and simulated luminosity functions and the number of post main sequence vs. total number of stars favours models with active star formation in the last $100 \mathrm{Myr}$. The data are best fitted by an IMF with Salpeter slope $(\alpha=2.35)$, and are also consistent with slopes in the range of $2 \lesssim \alpha \lesssim 2.6$. They exclude steeper IMF slopes. The mean star formation rate for a Salpeter IMF in the range of masses $0.6 \leq M \leq 100 M_{\odot}$ within the last $100 \mathrm{Myr}$ is $1.6 \times 10^{-4} M_{\odot} \mathrm{yr}^{-1} \mathrm{kpc}^{-2}$.
\end{abstract}

Key words. galaxies: elliptical and lenticular, $\mathrm{cD}$ - galaxies: stellar content - stars: fundamental parameters galaxies: individual: NGC 5128

\section{Introduction}

The two main traditional models of elliptical galaxy formation, the "monolithic collapse" (Partridge \& Peebles 1967) and the "hierarchical assembly" model (Toomre 1977) assume very different mechanisms and epochs of spheroid assembly. Correspondingly, they also predict different mean ages and metallicities for the stars that build these galaxies. Until the advent of the HST and $10 \mathrm{~m}$ class ground-based telescopes the studies of the stellar populations in the elliptical galaxies were restricted to integrated light photometry and spectroscopy. In the last few years, however, the nearest E/S0 galaxies have been resolved into stars (e.g. Soria et al. 1996; Davidge \& van den Bergh 2001; Schulte-Ladbeck et al. 2003). The study of the star formation histories of the resolved stellar populations observed in these galaxies is an important complementary approach to the studies of the high redshift Universe,

Send offprint requests to: $\mathrm{M}$. Rejkuba,

e-mail: mrejkuba@eso.org

* Based on observations collected at the European Southern Observatory, Paranal, Chile, within the Observing Programme 63. N-0229. aiming to address the questions of elliptical galaxy formation and evolution.

\subsection{Recent star formation in the halo of NGC 5128}

At the distance of 3.8 Mpc (Soria et al. 1996; Rejkuba 2004) NGC 5128 (=Centaurus A) is the dominant galaxy of the nearby Centaurus group and one of the closest giant elliptical galaxies to us. It is also one of the nearest AGNs and radio galaxies. It presents a number of peculiarities: the central dust lane with HII regions and OB associations at its edge, and the stellar shells which have presumably been formed in a recent accretion of a smaller companion galaxy (see review by Israel 1998). Moreover, young blue supergiants have been identified associated with the optical filaments in the north-eastern halo (Graham \& Price 1981; Graham 1998).

The young stars and $\mathrm{OB}$ associations in the halo of NGC 5128 are roughly aligned with the ionized gas and the radio jet (Morganti et al. 1999; Mould et al. 2000; Fassett \& Graham 2000; Rejkuba et al. 2001, 2002) over several kiloparsecs. The preferred mechanism for this recent star formation in the halo at $>14 \mathrm{kpc}$ away from the nucleus is through the 
direct interaction of the radio jet with the gas. The necessary amounts of neutral gas for this star formation have been detected in nearby fields through HI (Schiminovich et al. 1994) and CO molecular transitions (Charmandaris et al. 2000). Charmandaris et al. suggested that the molecular clouds in the distant halo are associated with stellar shells and are thus remains of the last accretion episode in which NGC 5128 has merged with a gas rich companion galaxy. While the gas could have just "hung around" in the galaxy halo, the presence of the radio jet triggered the star formation in highly collimated areas. The investigation of the star formation history of the young stars and the determination of the age spread in the young stellar population can constrain the formation of the radio jet or the time of the last accretion event. The metallicity of the gas of the accreted galaxy should be reflected in the newly formed stars.

The comparison of the optical color-magnitude diagrams (CMDs) with stellar evolutionary isochrones and young LMC clusters has indicated that these young stars can be as young as 10-15 Myr old (Mould et al. 2000; Fassett \& Graham 2000; Rejkuba et al. 2001). However, except for a comparison with one synthetic CMD (Mould et al. 2000), no detailed studies of the star formation history in the halo of NGC 5128 were made.

\subsection{The method}

Containing stars that were born during the whole life-time of the galaxy, CMDs represent snapshots of their star formation histories. Even when limited to the bright stars, the CMDs contain useful age and metallicity indicators: the colors of the red giant branch (RGB) stars are very sensitive to metallicity; the presence of bright asymptotic giant branch (AGB) stars signals an intermediate-age population; the colors and magnitudes of the bright main sequence (MS) stars and blue supergiants depend on the most recent star formation history, as well as on the metallicity.

The most effective way to analyse the distribution of stars in the CMD and to quantify the star formation history of a galaxy is through the comparison of the observed CMD with synthetic CMDs computed using stellar evolution models. This technique is based on the simulations of a large number of CMDs constructed assuming different star formation rates $(\operatorname{SFR}(t))$, chemical enrichment laws $(Z(t))$ and initial mass functions (IMF). The photometric errors and the incompleteness of the data are also simulated. Each simulated CMD then depends on all the main parameters which determine the distribution of stars in a CMD. In this way it is best accounted for the evolutionary behaviour of stars, small number of stars, and spread due to observational effects. The synthetic CMDs are then compared to the observed ones in order to determine the acceptable range of parameters and the best fitted models. This technique is currently used by several groups to investigate the star formation history (SFH) in dwarf galaxies (Ferraro et al. 1989; Tosi et al. 1991; Bertelli et al. 1992; Tolstoy 1996; Gallart et al. 1996a, 1996b; Dolphin 1997; Greggio et al. 1998; Hurley-Keller et al. 1998; Hernández et al. 1998;
Cole et al. 1999; Olsen 1999; Schulte-Ladbeck et al. 2000; Harris \& Zaritsky 2001).

Here we apply this technique to investigate the recent star formation history of the halo of NGC 5128. The observations were taken with VLT using FORS 1 and ISAAC in the $U, V$ and $K$ s bands. Their full description as well as data reduction and the resulting CMDs were presented in Rejkuba et al. (2001).

This paper starts with the description of the synthetic CMD simulator, including a detailed description of the algorithms used. In the next section the young stellar population in the north-eastern halo field is simulated. We test two different metallicities and a range of star formation histories. The initial mass function is also investigated.

\section{Description of the synthetic CMD simulator}

Our synthetic CMD simulator is developed based on the code used by Greggio et al. (1998). The construction of a synthetic CMD goes through the following steps:

1. Random extraction of mass according to the initial mass function (IMF), age according to a chosen star formation rate (SFR) law, and metallicity according to the chemical enrichment law $(Z(t))$.

2. Positioning of the extracted star as a function of mass, age, and metallicity on the theoretical HR diagram $\left(\log \left(L / L_{\odot}\right)\right.$, $\left.\log \left(T_{\text {eff }}\right)\right)$ interpolating within stellar evolutionary tracks.

3. Transformation of the theoretical position of the star in the HR diagram $\left(\log \left(L / L_{\odot}\right), \log \left(T_{\text {eff }}\right)\right)$ into the observational CMD assigning the bolometric corrections through interpolation within conversion tables.

4. Application of observational uncertainties, photometric errors and incompleteness, according to the results of artificial star experiments on the real data.

5. Iteration until a certain criterion (e.g. the total number of stars generated, the total mass extracted, or the total number of stars in a certain region of the simulated CMD) is satisfied.

The algorithms used for steps 1 and 2 are relatively straightforward, and used in a similar way by most groups. Hence we describe them in the Appendix. In the following sections we address steps 3 and 4 in more detail as the final results depend critically on them.

\subsection{Bolometric corrections}

Bolometric corrections (BCs) are needed to convert the theoretical $\log (L)-\log \left(T_{\text {eff }}\right)$ point into magnitude and color, to be compared with the data. An accurate knowledge of the photometric passbands is as important to synthetic photometry as the knowledge of the standard stars and the proper calibration procedure is to observational photometry. The calibration of our data was described in detail in Rejkuba et al. (2001). We have derived a full set of calibration equations including the color term for the optical photometry, while only the zero point was derived for the near-IR photometry. Thus our photometric 

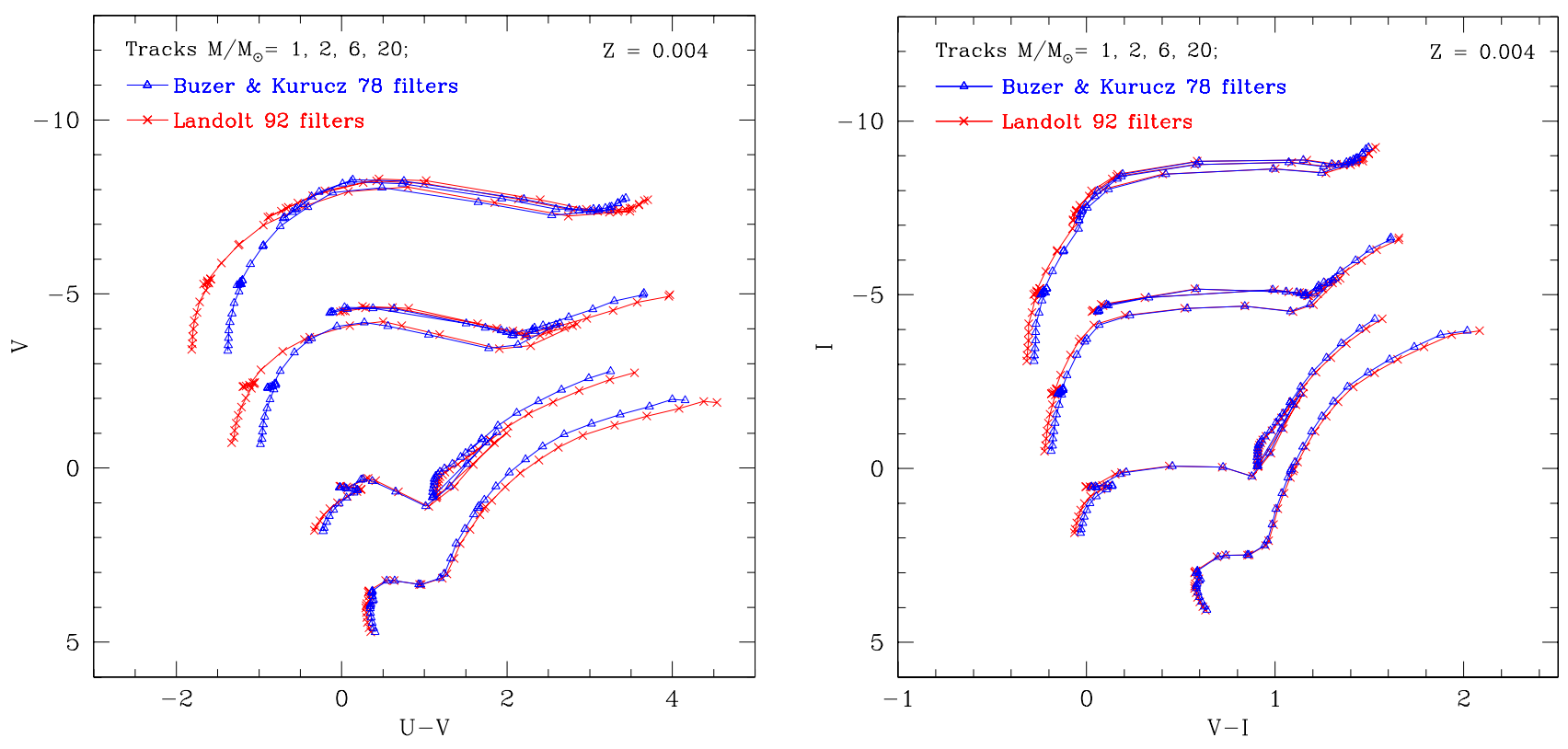

Fig. 1. Left: Fagotto et al. (1994) tracks for $Z=0.004$, transformed into the $V$ vs. $U-V$ plane by applying bolometric corrections obtained by convolving spectral energy distributions of Kurucz stellar atmospheres (see text) with the two sets of $U B V$ filters: Landolt (1992; red lines with crosses) and Buser \& Kurucz (1978; blue lines with triangles) The masses of the tracks are indicated. Right: the same as on the left, but for $I$ vs. $V-I$. The $V$ filter transmission curves are from Landolt (1992; red lines with crosses) and Buser \& Kurucz (1978; blue lines with triangles). The $I$ passband is the same in both cases.

system consists of Landolt (1992) $U$ and $V$ and ISAAC $K_{\mathrm{S}}$ (Cuby et al. 2003 ${ }^{1}$ ).

The Kurucz (1993) database of ATLAS9 synthetic spectra covers a large range in temperatures $\left(3500 \leq T_{\text {eff }} \leq 50000 \mathrm{~K}\right)$, and gravities $(0 \leq \log g \leq 5)$. Recently these models have been re-calculated by Castelli et al. (1997) and carefully compared to stellar data. Of the two sets in Castelli's grid, the one without overshooting is found to yield the best match to the observations (Bessell et al. 1998). Therefore we adopt the "NOVER" files at http : //kurucz . harvard . edu/ for metallicities $[\mathrm{M} / \mathrm{H}]=-2.0,-1.5,-1.0,-0.5,0.0$ and +0.5 , and microturbulent velocity $\xi=2 \mathrm{~km} \mathrm{~s}^{-1}$ to derive BCs as function of gravity, effective temperature, and metallicity. This is done by convolving the spectral energy distributions from the Kurucz stellar library with filter transmission curves $S_{\lambda}^{i}$ :

$$
\left\langle F_{i}\right\rangle=\frac{\int_{0}^{\infty} F_{\lambda}^{i} S_{\lambda}^{i} \mathrm{~d} \lambda}{\int_{0}^{\infty} S_{\lambda}^{i} \mathrm{~d} \lambda} .
$$

The magnitude and the bolometric correction are then by definition:

$$
\begin{aligned}
& M_{i}=-2.5 \log \left\langle F_{i}\right\rangle+Z P_{i} \\
& B C_{i}=M_{\mathrm{bol}}-M_{i}=-2.5 \log \left(\sigma T_{\mathrm{eff}}^{4}\right)+2.5 \log \left\langle F_{i}\right\rangle-Z P_{i} \\
& \text { where } \sigma T_{\mathrm{eff}}^{4}=\int_{0}^{\infty} F_{\lambda} \mathrm{d} \lambda .
\end{aligned}
$$

The calibration of the zero points in each band follows from the definition of the $V$-band bolometric correction of the Sun to be

\footnotetext{
${ }^{1}$ http://www.eso.org/instruments/isaac/index.html
}

$B C_{V}^{\odot}=-0.07$, and the colors of Vega $M_{V}^{\mathrm{Vega}}-M_{i}^{\mathrm{Vega}}=0$. The spectral energy distributions of the Sun and Vega were taken from the Kurucz library as well.

It is known that the Kurucz (1993) spectral energy distributions are not very accurate for cool stars due to inadequate molecular opacities, non-LTE, and sphericity related effects (e.g. Morossi et al. 1993). To extend our bolometric corrections to low temperatures $\left(T_{\text {eff }} \lesssim 4200 \mathrm{~K}\right)$, we used the empirically determined temperatures and bolometric corrections from Montegriffo et al. (1998). Montegriffo et al. do not have fully developed metallicity classes, but rather define metal-poor $([\mathrm{M} / \mathrm{H}] \leq-1.0 \mathrm{dex})$ and metal-rich $([\mathrm{M} / \mathrm{H}] \geq-1.0 \mathrm{dex})$ samples. The differences in bolometric corrections between the two groups span a wide range $0.05-0.4 \mathrm{mag} / \mathrm{dex}$. We also notice that their bolometric corrections are determined only for giant stars. However, the last issue is not a problem, since the low temperature stars in our case are giants with photometry in the $V$ and $K_{\mathrm{s}}$-bands. In the $U V$ only bright young stars are observed, the giants are too faint in the $U$-band.

We calculate the $\log g$ of each simulated star with ( $\left.\log M / M_{\odot}, \log L, T_{\text {eff }}\right)$ using the following two equations:

$R^{2}=\frac{L}{4 \pi \sigma T_{\mathrm{eff}}^{4}}$

$\log \left(g / g_{\odot}\right)=\log \left(M / M_{\odot}\right)-2 \log \left(R / R_{\odot}\right)$

where $\log g_{\odot}=4.437, \sigma=5.67 \times 10^{-5} \mathrm{erg} / \mathrm{cm}^{2} / \mathrm{K}^{4}, L_{\odot}=$ $3.826 \times 10^{33} \mathrm{erg} / \mathrm{s}$ and $R_{\odot}=6.9599 \times 10^{10} \mathrm{~cm}$. The linearly interpolated bolometric correction, as a function of $\log T_{\text {eff }}$ and $\log g$, is then applied to obtain magnitudes for the simulated stars.

In Fig. 1 we compare the evolutionary tracks of Fagotto et al. (1994) for $Z=0.004$ transformed using two different 


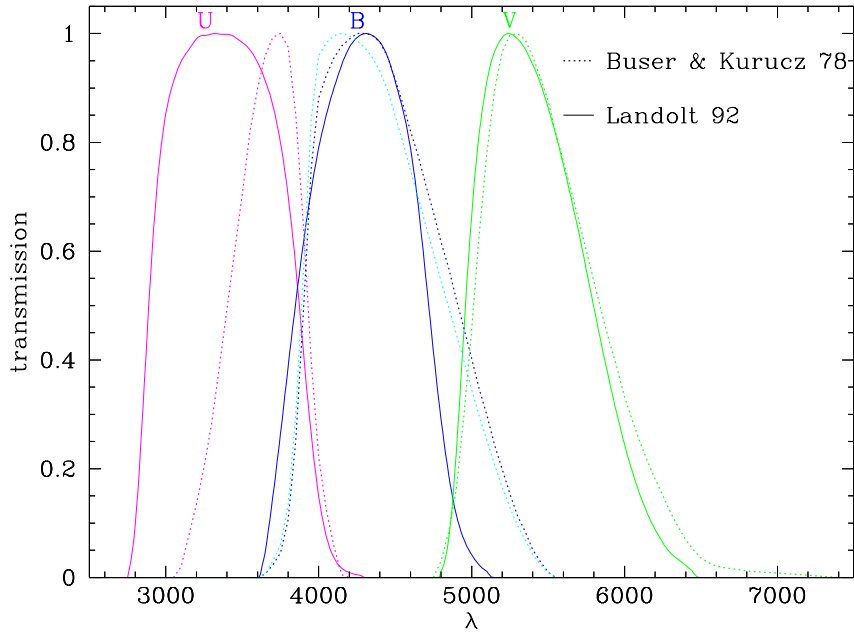

Fig. 2. Comparison of the Landolt (1992; full lines) and Buser \& Kurucz (1978; dotted lines) $U B V$ passbands.

sets of $U B V$ filters. In the right panel the comparison of $I$ vs. $V-I$ shows an excellent agreement of the colors of tracks for both sets of filters, but in the left panel it is obvious that our bolometric corrections calculated using the Landolt filters produce wider range of colors for the tracks in $U-V$. We investigated in detail the origin of this difference, finding that it is entirely due to different $U$ filter used in calculating the bolometric corrections.

The Padova group uses Buser \& Kurucz (1978) UBV transmission curves, while our photometry has been calibrated to the Landolt (1992) system. The comparison of the passbands of the two photometric systems is given in Fig. 2. Note the large difference in the $U$-passband wavelength coverage. The $U$-band is known to be the most difficult to reproduce due to the presence of the atmospheric cut-off, the presence of the Balmer break within that band, and the strong blanketing by metals (see Fig. 3). The differences between the $B$ and $V$-band filters are much smaller. Buser \& Kurucz (1978) $U B V$ system has two $B$ filters, $B_{2}$ and $B_{3}$. Both of these systems claim to be based on the Johnson \& Morgan (1953) standard system with Buser \& Kurucz $U-B$ and $B-V$ colors defined as follows:

$(U-B)_{\mathrm{syn}}=\left(U-B_{2}\right)-1.093$

$(B-V)_{\mathrm{syn}}=\left(B_{3}-V\right)+0.710$.

We have calculated the bolometric corrections by convolving the Kurucz stellar library with the $U B_{2} B_{3} V$ filters of Buser \& Kurucz (1978). There is an excellent agreement between the transformed tracks and Padova isochrones (Bertelli et al. 1994) if the following definition of the $U-V$ color is adopted:

$(U-V)_{\text {syn }}=\left(U-B_{2}\right)+\left(B_{3}-V\right)$.

The difference in the zero point is due to different calibration of the zero point bolometric corrections adopted by Buser \& Kurucz with respect to the Padova isochrones (Girardi et al. 2002). The Padova group adopted the standard calibration of the synthetic photometry which is based on the colors and magnitude of Vega, similar to our procedure.

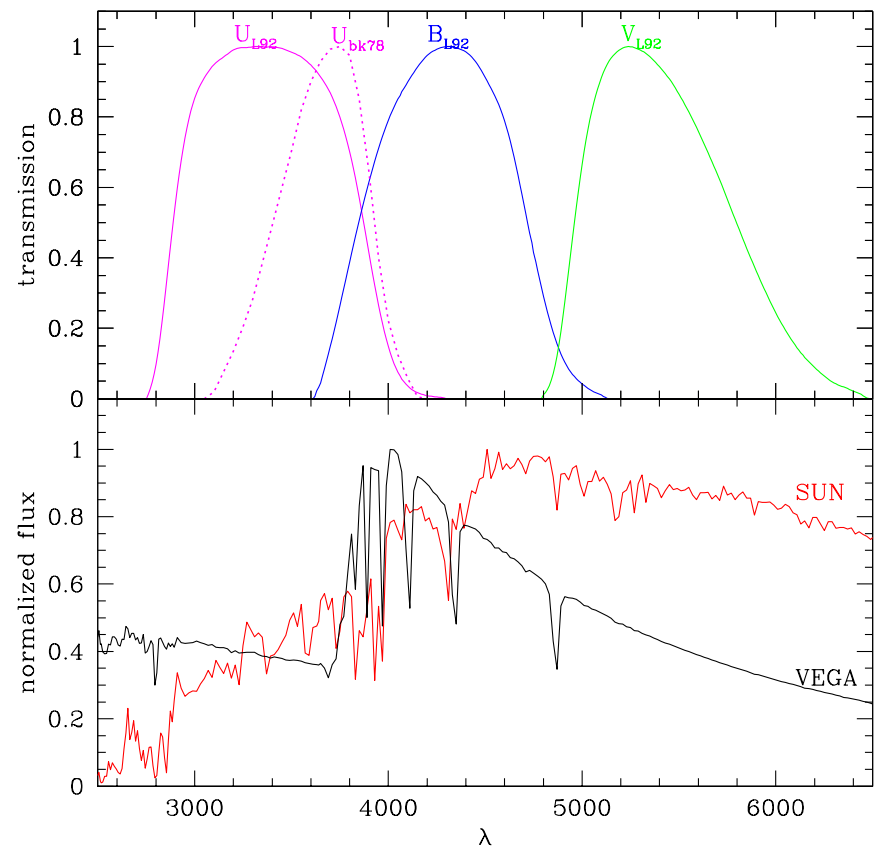

Fig. 3. The spectral energy distributions of the Sun and Vega are compared with the Landolt (1992) $U B V$ passbands (full lines) and Buser $\&$ Kurucz (1978) $U$ filter (dotted line).

This example shows how important it is to know precisely the transmission curves and the characteristics of the photometric system used. The calibration of the synthetic magnitude system has to be done in the same way as the data, by convolving the spectral energy distributions from the stellar library with the same filters used for the real observations.

Our data have been calibrated on Landolt's stellar catalogue (Landolt 1992), but observed with the FORS1 telescope+CCD+filter system, so that our system's throughput in the $U$ band is indeed much closer to the Bessel $U$-filter (with a passband similar to the Buser \& Kurucz 1978), mainly due to the CCD response curve. The best approach to calibrate our photometry would have been to use a standard system which matches the one used in the observations (Bessell 1995). Unfortunately, our list of observed standards has no star in common with Bessell's (1995) list.

The $U$-magnitude difference between the two systems depends on the star's spectral energy distribution, and therefore on temperature, gravity and metallicity. To a first approximation, the calibration equation corrects for this systematics as a function of the $(U-V)$ color. However, a residual systematic error exists, which stems from applying to all of the observed stars the calibration regression valid for the standard stars. In order to estimate the residual error we have calculated the $U$-magnitude difference in the two $U$ passbands of the complete grid of Kurucz models. The (FORS1 - Landolt) difference of the $U$ band Bolometric Corrections increases from $\sim-0.2$ to $\sim+0.4$, going from the hottest to the coolest models in the grid. Once the color equation applied to the data is taken into account, the residual difference ranges from $\sim-0.06$ mag for the blue stars $(U-V<0)$, to $\sim 0.25$ mag at $(U-V)>2$. We notice that this is the maximum residual error that we expect 
in our photometry, as it includes a very wide range of stellar parameters. Nevertheless, our photometry will tend to be too faint for the blue stars and too bright for the red stars. We will discuss in the last section the impact of this unceratinty on our results.

\subsection{Observational biases}

The synthetic CMD needs to be "corrected" for observational biases before comparing it with the observed CMD. There are two main types of observational biases:

- photometric errors: the magnitudes of stars are measured with some uncertainty;

- incompleteness: some stars are lost due to crowding or high background.

Blending is an additional bias that is important in crowded fields, and that combines properties of photometric errors and incompleteness.

We used the extensive artificial stars experiments (the full description can be found in Rejkuba et al. 2001) to assign the observational biases. In the artificial star experiments the input magnitude is given, and the output magnitude measured at each position of the input magnitude. If the star was lost in the background noise, or if it was completely blended with a much brighter object producing a magnitude difference between the input and the measured magnitude of more than 0.75 , we considered it to be non-detected. Observational biases are applied to magnitudes to which the appropriate distance modulus and extinction were added. Thus, when applying the observational biases we pick a random object from the input table of the artificial star experiments that has magnitude and color similar to the simulated star. The simulated star then receives a realistic photometric error (magnitude difference between the recovered and input magnitude in the artificial star experiment), and incompleteness (the star in the artificial star experiment was detected or not).

\section{Young stellar population observed in the UV CMD}

The observations of the young stars in the north-eastern shell field of NGC 5128 consist of pairs of $U$ and $V$-band images taken at ESO Paranal observatory UT1 Very Large Telescope with FORS1 instrument (Szeifert 2002 $2^{2}$ The photometry is $50 \%$ complete for magnitudes brighter than $V=27.5$ and $U=25$ (Rejkuba et al. 2001). The shallower $U$-band photometry drives the incompleteness and the photometric errors in the $U V$ CMD (Fig. 4) and thus limits our analysis to magnitudes brighter than $V \sim 25.5$.

In Fig. 4 we display the $V$ vs. $U-V$ CMDs of this field with different values of DAOPHOT errors in both $U$ and $V$ applied as a selection criterion. The vertical blue sequence at $U-V \sim-1$ is populated with massive MS stars in NGC 5128, while most of the red stars belong to the foreground Galactic

\footnotetext{
${ }^{2}$ http://www.eso.org/instruments/fors/userman/
}
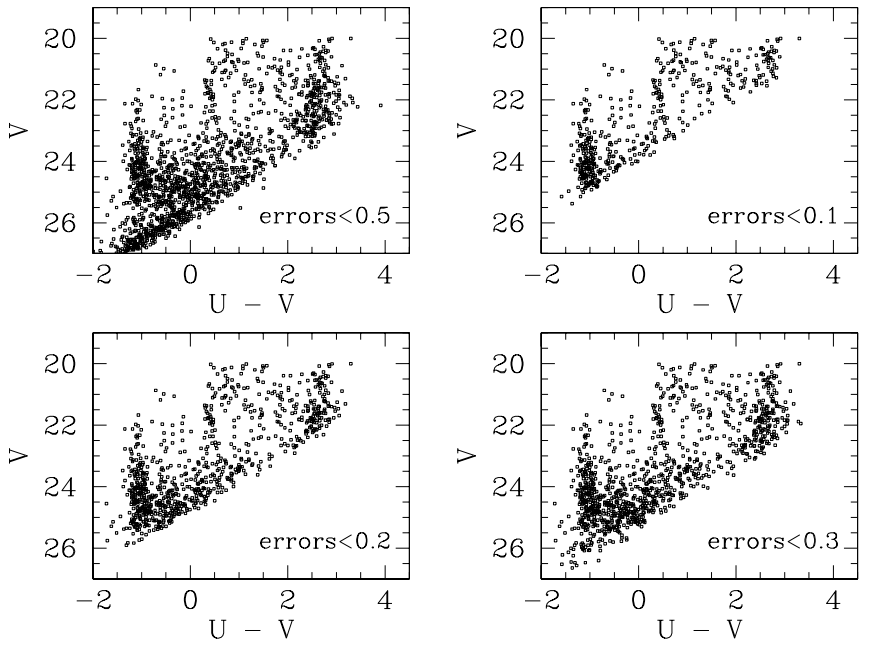

Fig. 4. Color-magnitude diagrams of the young stellar populations in the shell field of NGC 5128 with different cuts applied on the size of the photometric errors in both filters $U$ and $V$.

Table 1. Fraction of stars in different parts of observed CMD - all stars, blue $(U-V<0)$, intermediate color $(0<U-V<1.5)$, and red stars $(U-V>1.5)$ - that survive the error selection at maximum error of $0.1,0.2$, and 0.3 , with respect to selection with $\sigma<0.5$. Only the stars with $U$-band completeness limits larger than $50 \%$ are selected, corresponding to $V$-band magnitudes smaller than 25.5 in the bluest part of the CMD.

\begin{tabular}{ccccc}
\hline \hline$\sigma$ cut & all stars & blue & intermediate & red \\
\hline$\sigma<0.3$ & $81 \%$ & $97 \%$ & $70 \%$ & $75 \%$ \\
$\sigma<0.2$ & $66 \%$ & $87 \%$ & $53 \%$ & $55 \%$ \\
$\sigma<0.1$ & $37 \%$ & $52 \%$ & $29 \%$ & $26 \%$ \\
\hline
\end{tabular}

population. The diagonal cut-off at faint magnitudes is a consequence of the $U$-band incompleteness and the sharp bright magnitude cut-off at $V=20$ is due to the saturation limit of the $V$-band images. The CMD with all stars that had $\sigma<0.5$ is presented in the upper left panel. Comparing it with the other panels it is evident that many of the red stars have large photometric errors. This is clearer in Table 1, where we list fraction of stars that remain in the CMD when a cut is applied at $\sigma$ of $0.3,0.2$ and 0.1 . Even when only the stars with the smallest photometric errors are selected, more than $50 \%$ of the blue stars survive and the young blue sequence remains well populated. We shall use this feature to investigate the recent star formation history in this field.

Figure 5 shows the observed CMD with the transformed Padova tracks superimposed, for $Z=0.004$ (left) and $Z=$ 0.008 (right). No spectroscopic estimate of the metallicity of the young stars in Cen $\mathrm{A}$ is available in the literature. The two considered $\mathrm{Z}$ values are motivated by the Rejkuba et al. (2001) estimate, which is based on the best fitting isochrones. Notice, however, that due to the discussed difference between the Landolt and the Buser $U$ filter, the Rejkuba et al. estimate needs to be reconsidered with the appropriately transformed tracks. A distance modulus of $27.8 \mathrm{mag}$ (Soria et al. 1996) in combination with a color excess of $E(U-V)=0.62$ yields a good fit to the position of the MS stars in Fig. 5. According to 

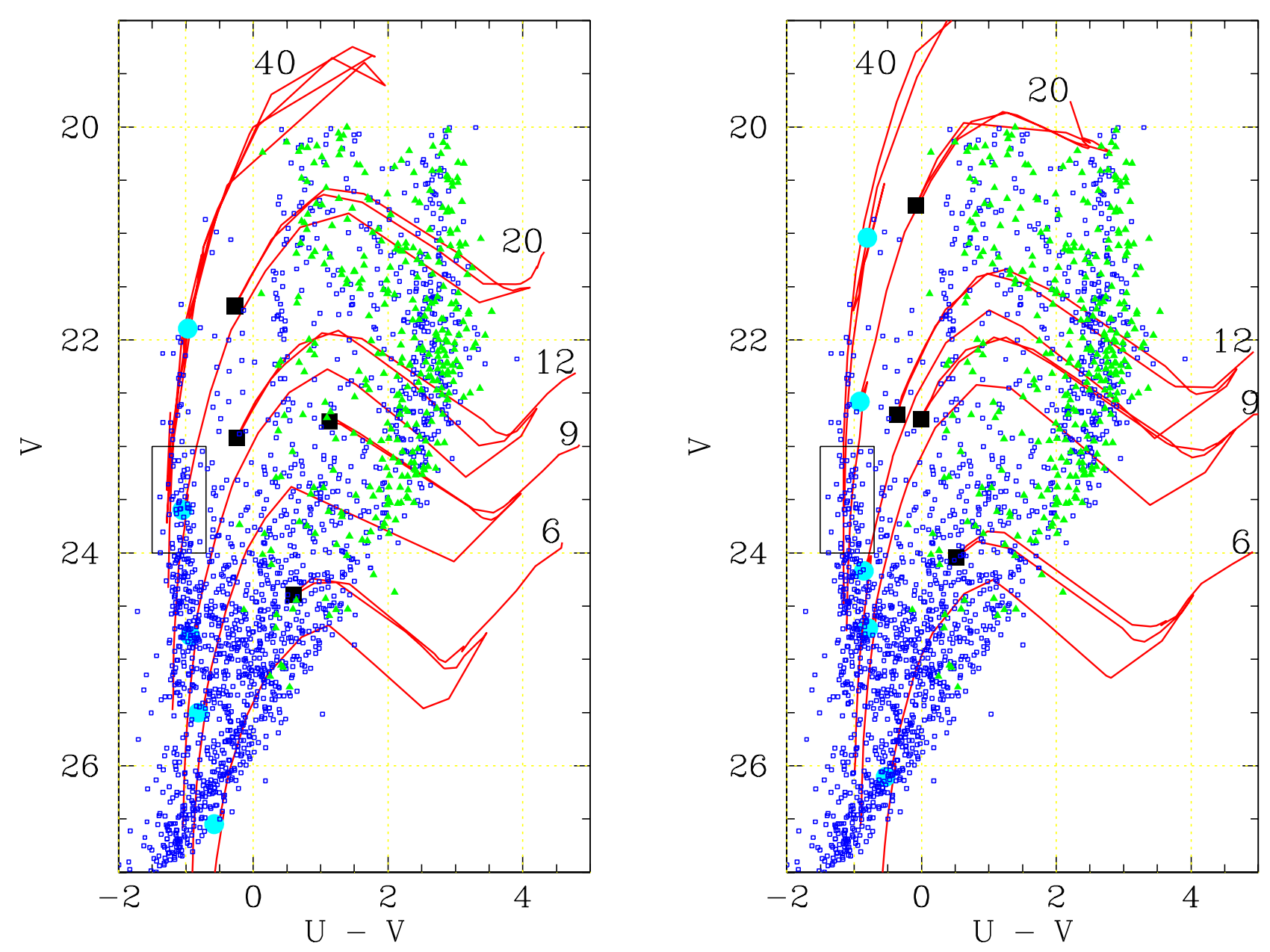

Fig. 5. Comparison of the the $U V$ CMD with stellar evolutionary tracks for $Z=0.004$ (left) and $Z=0.008$ (right panel) and masses of 6 , 9 , 12,20 and $40 M_{\odot}$. Overplotted are also the expected foreground stars (green filled triangles) from the Besançon simulations (see Rejkuba et al. 2001). Along the line connecting MS turn-offs (large cyan dots) and along the line connecting blue edges (large black squares) most of the stars are expected according to the models. A box on the main sequence between magnitudes 23 and 24 indicates the area used to constrain the simulations.

the Cardelli et al. (1989) extinction curve, $E(U-V)=0.62$ implies $E(B-V)=0.35$, which is much larger than the foreground extinction $E(B-V)=0.11$ from Schlegel et al. (1998) map, and implies a substantial amount of internal (perhaps differential) reddening. Rejkuba et al. estimated $E(B-V)=0.15$, due to the different $U$ filter. Choosing a somewhat larger distance modulus of 27.92 mag (Harris et al. 1999; Rejkuba 2004) does not change our conclusions significantly.

The magnitudes and colors of the stars belonging to the Milky Way, that are expected to be found in a 6.8 $\times 6.8$ field of view of FORS1, at $l=309.5^{\circ}, b=19.5^{\circ}$, have been simulated using Besançon galactic model of stellar populations (Robin \& Creze 1986; Robin et al. 1996). Realistic photometric errors and incompleteness has been applied through crowding simulations. In total, at least 340 stars with colors $U-V>0$ are expected to belong to the foreground Milky Way population (see Rejkuba et al. 2001). They are overplotted over the observed CMD in Fig. 5 as (green) filled triangles. Only a small number of stars with $(U-V)>0$ are expected to be blue and red supergiants evolving along the blue loops in the NGC 5128 halo. Unfortunately, the width of the blue loops cannot be used to constrain the metallicity both because of the huge amount of the foreground contamination and, because of the uncertain extension of the blue loops (Ritossa 1996).

The location of the post main sequence (PMS) stars, blue and red supergiants, and the extension of the loops depends critically on the convection criteria or the mixing efficiency, the metallicity, the opacities at intermediate temperatures, the mass loss and the overshooting (Renzini et al. 1992; Ritossa 1996). At larger metallicities the thermal conductivity of the envelope is lower leading to a larger thermal imbalance of the stellar envelope and to a faster expansion to the red. The more metalrich models have thus redder red giant minima. The models that adopt efficient mixing in the semiconvective region during the hydrogen shell burning spend most of the PMS life-times as blue supergiants, while the less efficient mixing drives the runaway expansion toward the red (Ritossa 1996). The convective core overshoot during the MS in combination with efficient mixing can, however, produce models with wider loops (e.g. Fagotto et al. 1994).

The overshooting and opacity, constraining $T_{\text {eff }}$, drive the amplitude of blue loops and, through the dependence of 
Table 2. Main sequence and total life-times of the stars with masses larger than $4 M_{\odot}$ and metallicities $Z=0.004$ and $Z=0.008$ (Fagotto et al. 1994).

\begin{tabular}{|c|c|c|c|c|}
\hline \multirow[t]{2}{*}{ 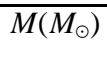 } & $\overline{\tau_{\mathrm{MS}}(\mathrm{Myr})}$ & $\overline{\tau_{\text {tot }}(\mathrm{Myr})}$ & $\overline{\tau_{\mathrm{MS}}(\mathrm{Myr})}$ & $\overline{\tau_{\text {tot }}(\mathrm{Myr})}$ \\
\hline & \multicolumn{2}{|c|}{$Z=0.004$} & \multicolumn{2}{|c|}{$Z=0.008$} \\
\hline 5.0 & 101.89 & 112.09 & 104.91 & 116.05 \\
\hline 6.0 & 70.98 & 76.74 & 71.63 & 77.71 \\
\hline 7.0 & 52.58 & 56.39 & 52.40 & 56.26 \\
\hline 9.0 & 33.07 & 35.25 & 32.42 & 34.52 \\
\hline 12.0 & 20.20 & 21.48 & 19.85 & 21.08 \\
\hline 15.0 & 14.35 & 15.27 & 14.03 & 14.93 \\
\hline 20.0 & 9.805 & 10.48 & 9.44 & 10.10 \\
\hline 30.0 & 6.37 & 6.86 & 6.18 & 6.66 \\
\hline 40.0 & 5.01 & 5.44 & 4.89 & 5.31 \\
\hline 60.0 & 3.85 & 4.20 & 3.84 & 4.23 \\
\hline 100.0 & 3.11 & 3.38 & 3.12 & 3.44 \\
\hline 120.0 & 2.98 & 3.24 & 3.01 & 3.33 \\
\hline
\end{tabular}

bolometric corrections on the effective temperature $\left(\mathrm{BC}\left(T_{\mathrm{eff}}\right)\right)$, they produce the sharp edge in the observed CMD. In particular the $9 M_{\odot}$ model for $Z=0.004$ has a very short loop (see Fig. 5). The blue excursion of this model is terminated at low temperatures, producing a sharp "red hook" in the simulated CMD (see below).

The extremely short evolutionary life-time for stars between the MS turn-off and blue edge leaves a "hole" in the CMD. On the other hand this area $(-0.5 \lesssim U-V \lesssim 0$ and $V \gtrsim 23$ ) of the observed CMD is populated. The possible reasons include different metallicity, inadequate input physics (combination of overshooting and opacity parameters), differential reddening, bolometric corrections, photometric errors and blends of several lower mass stars. Thus we expect not to be able to reproduce well this zone.

The evolutionary lifetimes of the plotted tracks are listed in Table 2. Comparing the tracks to the data we see that the oldest MS stars in our observed CMD are $\sim 40-50$ Myr old for the $Z=0.004$ and $\sim 50-65 \mathrm{Myr}$ for the $Z=0.008$ models. The faintest evolved stars are somewhat older ( $\sim 80 \mathrm{Myr})$, but buried in the foreground contamination. We can thus derive the most recent star formation, and check the IMF for stellar masses larger than $7 M_{\odot}$ from the bluest stars in the CMD.

Our simulations are constructed under the requirement of reproducing the stellar counts in a box on the MS, which contains 57 stars with $23 \leq V \leq 24$ and $U-V<-0.7$ (Fig. 5). The faintest stars in this box are $15 \mathrm{Myr}$ old. Therefore, any SFH with the last star formation episode ceasing earlier than 15 Myr ago would not populate this box, and we do not consider these models. Given the short age range sampled by the blue stars, and given their relatively smooth distribution, we consider only episodes with constant SFR, and vary the starting $\left(\tau_{\max }\right)$ and ending $\left(\tau_{\min }\right)$ epochs of active SF. We compare the synthetic and observed CMDs directly as well as the luminosity function along the MS for the stars brighter than $V=26$, which are not affected critically by photometric errors and incompleteness. In the next sections we address the following questions: "How old are the oldest and the youngest stars in the observed $U V$ CMD?" and "What is the slope of the initial mass function?"

\subsection{Age of the young stellar population in the NGC 5128 halo field}

The range of models probed is listed in Table 3. In the first column there is the model name, and in Cols. 2-4 the input parameters regarding the IMF and the duration of the star formation are given. The output total extracted mass in the range of 0.6 to $100 M_{\odot}$, the mean SFR for the same mass range, and the explored interval of the star formation $(\Delta \tau)$, as well as the total number of stars above the $80 \%$ incompleteness limit in $V$-band $(V<25.5)$, and the number of simulated blue plume stars $(U-V<-0.7$ for $V<25.5)$ are listed in Cols. 6-9 for $Z=0.004$, and $10,11,12$ and 13 for models using evolutionary tracks for metallicity $Z=0.008$, respectively. Due to the small range of ages and to the smooth appearance of the MS, a constant star formation was considered. Therefore the Cols. 6 and 10 list:

$\langle S F R\rangle=\frac{M_{\text {star }}}{\Delta \tau}$.

Four different IMF slopes have been used in the simulations. The dependence of the simulations on the IMF slope is discussed in detail in the next section. First we illustrate in detail the model CMDs made with Salpeter IMF ( $\alpha=2.35$ ), varying the age range, for the two metallicities: $Z=0.004$ (Fig. 6) and $Z=0.008$ (Fig. 7). The duration of the star formation episode is indicated in each panel as the starting-ending time. An ending time of 0 Myr means that the star formation is on-going.

The sharp blue edge of the blue loops is due to the theoretical form of the blue loops. In particular, the early termination of the $9 M_{\odot}$ model for $Z=0.004$ produces a bend in the simulated CMDs. This transition is much smoother for the $Z=0.008$ models. The appearance of the CMD suggests that the $9 M_{\odot}$ model for $Z=0.004$ should have bluer blue edge. The high degree of contamination prevents us to draw a robust conclusion, but the $U-V$ vs. $V$ CMD seems particularly suited to constrain the shape of the loops, and could be applied in stellar systems with spectroscopic abundance determination.

As the star formation lasts longer it is possible to observe the excursions of the stars in PMS phases. The reddest parts of the CMD, however, remain very sparsely populated due to the much shorter evolutionary timescales in the red with respect to the blue. Less massive $\left(M \lesssim 15 M_{\odot}\right)$ red supergiants evolve less rapidly. Few of them are visible in the upper red part of the simulated CMDs in which star formation was not terminated. Also, as the star formation gets longer, the MS gets populated with stars of smaller masses, the total number of which is governed by the IMF.

In the direct comparison of the simulated and observed CMDs, and the corresponding luminosity functions (Figs. 6 and 7) it can be seen that all the simulations with on-going star formation fit the data equivalently well irrespectively of the maximum stellar age $\left(\tau_{\max }\right)$. The luminosity function peak is best fitted with models with longer $\tau_{\max }(\gtrsim 30-50 \mathrm{Myr})$, but the differences between various models are within the Poissonian 
Table 3. Input parameters and results of the simulated star formation histories of young stellar populations. Columns 1 to 4 are identifier of the model, slope of the IMF, start and end of the star formation burst in Myr, respectively. In the next 8 columns extracted mass, mean star formation rate in the interval $\Delta \tau$, the total number $\left(N_{\text {tot }}\right)$ and the number of blue plume stars $\left(N_{\text {blue }}\right)$ in the simulated CMDs are given for $Z=0.004$ and $Z=0.008$.

\begin{tabular}{|c|c|c|c|c|c|c|c|c|c|c|c|}
\hline \multirow{3}{*}{$\begin{array}{l}\text { model } \\
\text { ID }\end{array}$} & \multirow{3}{*}{$\begin{array}{c}\text { IMF } \\
\alpha\end{array}$} & \multirow{3}{*}{$\begin{array}{c}\tau_{\max } \\
10^{6} \mathrm{yr}\end{array}$} & \multirow{3}{*}{$\begin{array}{c}\tau_{\min } \\
10^{6} \mathrm{yr}\end{array}$} & \multicolumn{4}{|c|}{$Z=0.004$} & \multicolumn{4}{|c|}{$Z=0.008$} \\
\hline & & & & mass & $\langle S F R\rangle$ & $N_{\text {tot }}$ & $N_{\text {blue }}$ & mass & $\langle\langle S F R\rangle$ & $N_{\text {tot }}$ & $N_{\text {blue }}$ \\
\hline & & & & $10^{6} M_{\odot}$ & $M_{\odot} / \mathrm{yr}$ & & & $10^{6} M_{\odot}$ & $M_{\odot} / \mathrm{yr}$ & & \\
\hline salp01 & 2.35 & 1000 & 0 & 6.79 & 0.007 & 512 & 276 & 6.44 & 0.006 & 499 & 279 \\
\hline salp02 & 2.35 & 500 & 0 & 3.97 & 0.008 & 551 & 296 & 3.38 & 0.007 & 472 & 257 \\
\hline salp03 & 2.35 & 200 & 0 & 1.38 & 0.007 & 496 & 289 & 1.47 & 0.007 & 546 & 288 \\
\hline salp04 & 2.35 & 100 & 0 & 0.66 & 0.007 & 460 & 256 & 0.61 & 0.006 & 460 & 250 \\
\hline salp05 & 2.35 & 65 & 0 & 0.44 & 0.007 & 415 & 259 & 0.35 & 0.005 & 368 & 225 \\
\hline salp06 & 2.35 & 50 & 0 & 0.29 & 0.006 & 351 & 224 & 0.31 & 0.006 & 377 & 226 \\
\hline salp07 & 2.35 & 30 & 0 & 0.23 & 0.008 & 430 & 306 & 0.19 & 0.006 & 345 & 229 \\
\hline salp08 & 2.35 & 10 & 0 & 0.08 & 0.008 & 273 & 219 & 0.08 & 0.008 & 283 & 241 \\
\hline salp09 & 2.35 & 5 & 0 & 0.07 & 0.014 & 254 & 216 & 0.07 & 0.014 & 278 & 239 \\
\hline salp10 & 2.35 & 100 & 10 & 7.73 & 0.086 & 3080 & 887 & 6.43 & 0.071 & 2685 & 801 \\
\hline salp11 & 2.35 & 100 & 5 & 1.52 & 0.016 & 816 & 353 & 1.40 & 0.015 & 806 & 339 \\
\hline salp12 & 2.35 & 100 & 2.5 & 0.90 & 0.009 & 568 & 273 & 0.94 & 0.010 & 633 & 313 \\
\hline scal01 & 2.60 & 1000 & 0 & 12.8 & 0.013 & 591 & 288 & 9.95 & 0.010 & 478 & 257 \\
\hline scal02 & 2.60 & 500 & 0 & 6.65 & 0.013 & 641 & 291 & 5.38 & 0.011 & 548 & 261 \\
\hline scal03 & 2.60 & 200 & 0 & 2.30 & 0.011 & 610 & 301 & 2.12 & 0.011 & 526 & 254 \\
\hline scal04 & 2.60 & 100 & 0 & 1.65 & 0.017 & 719 & 337 & 1.19 & 0.012 & 600 & 296 \\
\hline scal05 & 2.60 & 65 & 0 & 0.96 & 0.015 & 619 & 349 & 0.96 & 0.015 & 704 & 372 \\
\hline scal06 & 2.60 & 50 & 0 & 0.65 & 0.013 & 512 & 289 & 0.57 & 0.011 & 492 & 296 \\
\hline scal07 & 2.60 & 30 & 0 & 0.32 & 0.011 & 406 & 267 & 0.32 & 0.011 & 418 & 267 \\
\hline scal08 & 2.60 & 10 & 0 & 0.14 & 0.014 & 253 & 206 & 0.10 & 0.010 & 191 & 160 \\
\hline scal09 & 2.60 & 5 & 0 & 0.15 & 0.030 & 291 & 257 & 0.12 & 0.025 & 257 & 217 \\
\hline scal10 & 2.60 & 100 & 10 & 12.1 & 0.134 & 3497 & 921 & 10.0 & 0.112 & 3030 & 777 \\
\hline scal11 & 2.60 & 100 & 5 & 1.95 & 0.020 & 789 & 314 & 2.09 & 0.022 & 787 & 309 \\
\hline scal12 & 2.60 & 100 & 2.5 & 2.43 & 0.025 & 984 & 442 & 1.49 & 0.015 & 669 & 289 \\
\hline $\operatorname{imf} 301$ & 3.00 & 1000 & 0 & 34.8 & 0.035 & 939 & 366 & 28.4 & 0.028 & 743 & 314 \\
\hline imf302 & 3.00 & 500 & 0 & 21.6 & 0.043 & 1038 & 419 & 18.4 & 0.037 & 819 & 344 \\
\hline $\operatorname{imf} 303$ & 3.00 & 200 & 0 & 6.93 & 0.035 & 819 & 312 & 7.54 & 0.038 & 891 & 378 \\
\hline imf304 & 3.00 & 100 & 0 & 4.41 & 0.044 & 991 & 426 & 3.62 & 0.036 & 792 & 319 \\
\hline imf305 & 3.00 & 65 & 0 & 2.51 & 0.039 & 693 & 340 & 1.82 & 0.028 & 573 & 278 \\
\hline imf306 & 3.00 & 50 & 0 & 2.63 & 0.053 & 869 & 464 & 1.87 & 0.037 & 738 & 361 \\
\hline imf307 & 3.00 & 30 & 0 & 1.29 & 0.043 & 601 & 363 & 0.95 & 0.032 & 503 & 309 \\
\hline $\operatorname{imf} 308$ & 3.00 & 10 & 0 & 0.49 & 0.049 & 304 & 238 & 0.55 & 0.055 & 362 & 278 \\
\hline imf309 & 3.00 & 5 & 0 & 0.50 & 0.101 & 331 & 277 & 0.39 & 0.078 & 287 & 237 \\
\hline $\operatorname{imf} 310$ & 3.00 & 100 & 10 & 31.8 & 0.353 & 5756 & 1333 & 19.7 & 0.219 & 3267 & 784 \\
\hline $\operatorname{imf} 311$ & 3.00 & 100 & 5 & 6.02 & 0.063 & 1118 & 379 & 5.39 & 0.057 & 1082 & 396 \\
\hline $\operatorname{imf} 312$ & 3.00 & 100 & 2.5 & 5.22 & 0.053 & 1057 & 387 & 4.54 & 0.047 & 927 & 359 \\
\hline $\operatorname{imf} 201$ & 2.00 & 1000 & 0 & 3.87 & 0.004 & 402 & 239 & 3.75 & 0.003 & 388 & 235 \\
\hline $\operatorname{imf} 202$ & 2.00 & 500 & 0 & 2.11 & 0.004 & 433 & 258 & 1.68 & 0.003 & 370 & 231 \\
\hline $\operatorname{imf} 203$ & 2.00 & 200 & 0 & 0.93 & 0.005 & 490 & 271 & 0.68 & 0.003 & 358 & 222 \\
\hline $\operatorname{imf} 204$ & 2.00 & 100 & 0 & 0.40 & 0.004 & 377 & 237 & 0.43 & 0.004 & 445 & 261 \\
\hline $\operatorname{imf} 205$ & 2.00 & 65 & 0 & 0.32 & 0.005 & 486 & 309 & 0.26 & 0.004 & 386 & 248 \\
\hline imf206 & 2.00 & 50 & 0 & 0.19 & 0.004 & 362 & 256 & 0.20 & 0.004 & 411 & 260 \\
\hline $\operatorname{imf} 207$ & 2.00 & 30 & 0 & 0.10 & 0.003 & 312 & 233 & 0.14 & 0.005 & 419 & 290 \\
\hline imf208 & 2.00 & 10 & 0 & 0.05 & 0.005 & 252 & 215 & 0.03 & 0.003 & 193 & 165 \\
\hline $\operatorname{imf} 209$ & 2.00 & 5 & 0 & 0.03 & 0.005 & 190 & 173 & 0.03 & 0.007 & 232 & 212 \\
\hline $\operatorname{imf} 210$ & 2.00 & 100 & 10 & 4.25 & 0.047 & 2127 & 699 & 3.05 & 0.034 & 1705 & 517 \\
\hline $\operatorname{imf} 211$ & 2.00 & 100 & 5 & 0.99 & 0.010 & 693 & 318 & 0.87 & 0.009 & 625 & 311 \\
\hline $\operatorname{imf} 212$ & 2.00 & 100 & 2.5 & 0.52 & 0.005 & 445 & 253 & 0.45 & 0.004 & 422 & 235 \\
\hline
\end{tabular}



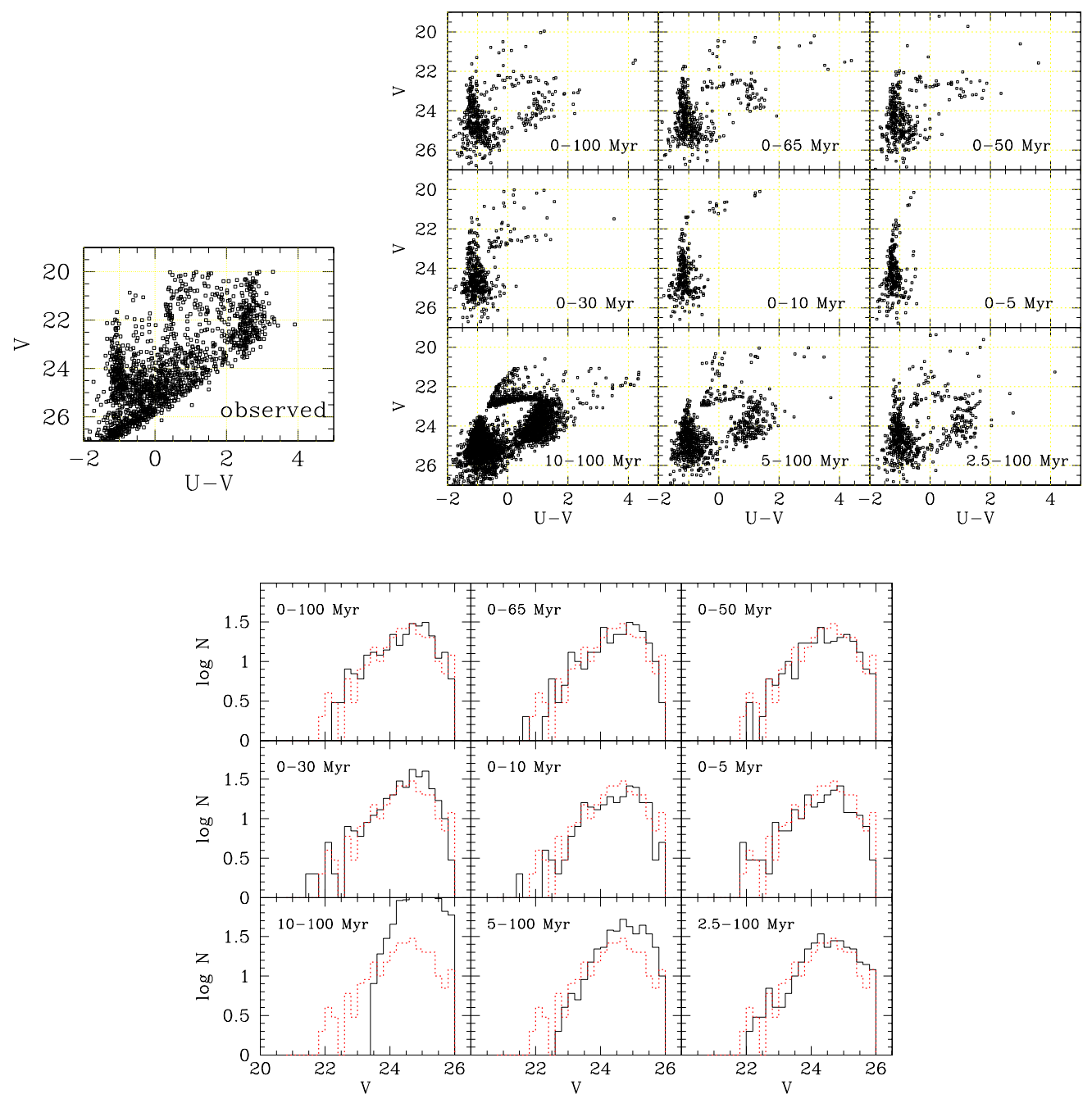

Fig. 6. Synthetic CMDs for $Z=0.004$ using the Salpeter IMF ( $\alpha=2.35$ ), the appropriate distance, and a constant SFR in the interval that is indicated in each panel (beginning-end of the burst). For comparison the observed CMD is also displayed. In the lower part simulated luminosity functions (solid lines) are compared with the observed luminosity functions (dotted lines). In the observed and simulated luminosity functions the color and magnitude cuts are applied, and only stars bluer than $(U-V)=-0.7$ and brighter than $V=26$ are compared.

errors. Hence, using only MS stars $(U-V<-0.7)$ it is not possible to constrain $\tau_{\max }$.

On the other hand, the simulations in which the star formation has stopped 5 or 10 million years ago, have many more stars in the lower MS and around the blue edge of the loops than observed. In order to populate the box on the MS between magnitudes 23 and 24, which contains the youngest MS stars (younger than 14-15 Myr) and thus the ones with the shortest evolutionary timescales, many more lower mass stars were produced in these simulations. Stopping the SF at 2.5 Myr produces a MS luminosity function in agreement with the observations.

In the left panels of Fig. 8 we plot the number of stars in the blue plume for all the models with $Z=0.008$ (top) and $Z=0.004$ (bottom). The observed value is shown as a green star. Different symbols are used for models with different IMF slopes as indicated in the legend. The dashed lines indicate the $3 \sigma$ Poissonian deviation from the observed value to which the random errors of the models have been added in quadrature. The models outside of the dashed lines in this figure are ruled out. So, the total number of MS stars and their luminosity function constrain $\tau_{\min }$ to be at most $2.5 \mathrm{Myr}$. The impact of the IMF on this constraint is discussed in the next section.

Although PMS stars cannot be used to select the best fitting model due to large uncertainties as discussed above, in the observed CMD there are 17 bright $(21<V<23)$ and blue stars $(-0.7<U-V<0)$ most probably in the PMS phases. They are found in the area of the CMD where there is no contamination by foreground Galactic stars. The models that have active star formation only over the last 5-10 Myr, have one or no stars in this area of the CMD. Thus, the star formation in this field must have been going on for more than $10 \mathrm{Myr}$.

Better limits to the duration of the star formation can be obtained by comparison between the number of stars in the MS and in the PMS phases. When comparing the observed and the simulated number of stars in the red part of the CMD, it is necessary to subtract the expected number of foreground stars according to the Galactic models. The ratio of the blue plume $(U-V<-0.7)$, and the total number of stars versus the duration of the star formation for the models described in Table 3 is 

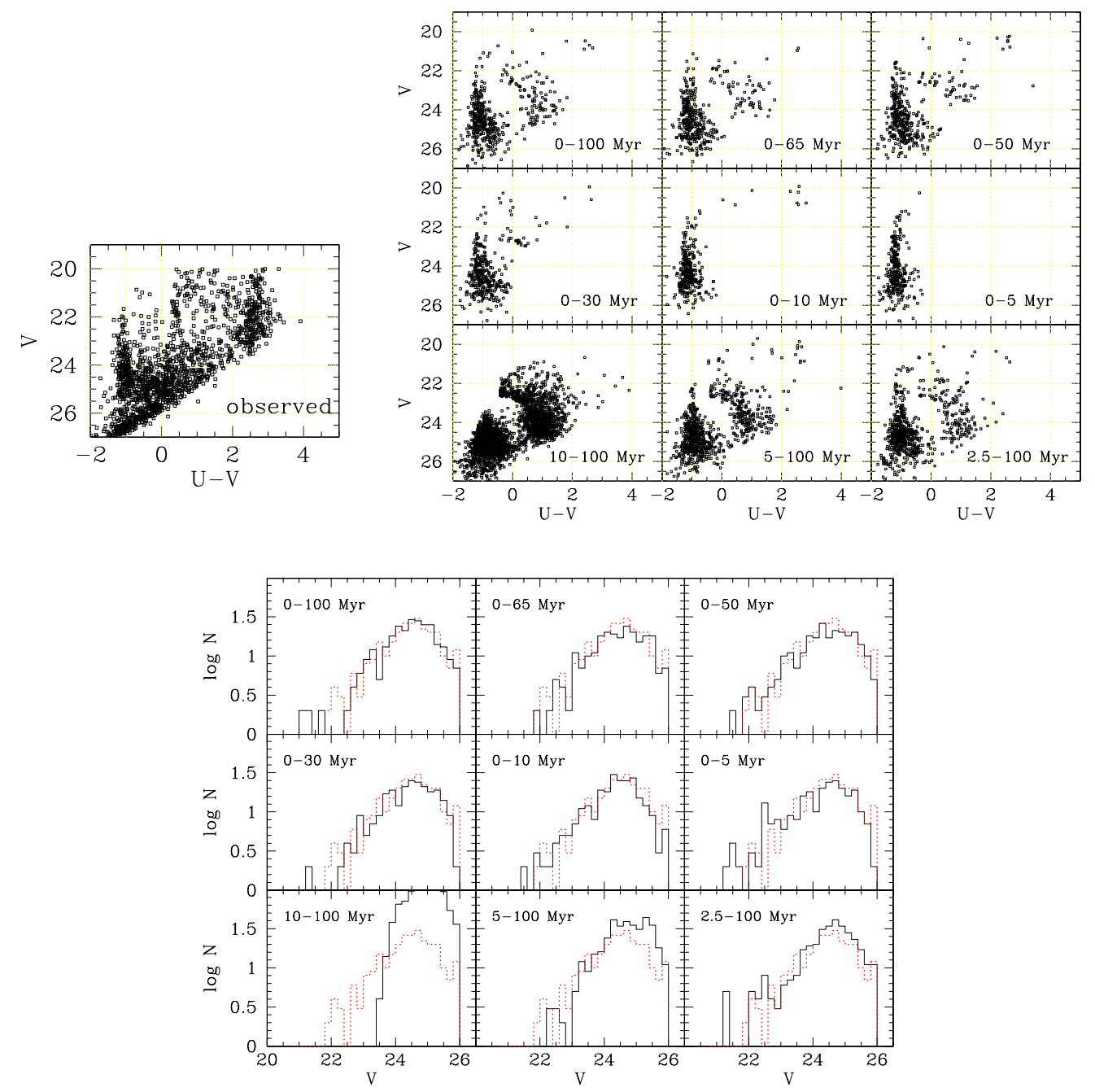

Fig. 7. Synthetic CMDs for $Z=0.008$ using the Salpeter IMF $(\alpha=2.35)$, the appropriate distance, and a constant SFR in the interval that is indicated in each panel (beginning-end of the burst). For comparison the observed CMD is also displayed on the left. In the lower panels the simulated luminosity functions (solid lines) are compared with the observed luminosity functions (dotted lines). In the observed and simulated luminosity functions the color and magnitude cuts are applied, and only stars bluer than $(U-V)=-0.7$ and brighter than $V=26$ are compared.

presented in the upper right panel of the Fig. 8 for $Z=0.008$, and lower right panel for $Z=0.004$ models. This ratio decreases with increasing $\tau_{\max }$, because the PMS phases become more populated.

The inflexion point in these diagrams corresponds to the maximum look-back time and hence the maximum age of the burst $\tau_{\max }$. It is $\sim 100 \mathrm{Myr}$ for the models with $Z=0.008$ and $\sim 90 \mathrm{Myr}$ for the $Z=0.004$ models. Of course, this value is the lower limit for the duration of the star formation. The older stars are not observed due to the incompleteness in the $U$-band, hence the ratio of the blue vs. total number of stars becomes constant for longer star formation durations.

The observed ratio of the blue vs. total number of stars in NGC 5128, which has been corrected for the expected amount of foreground contamination, is indicated with a star in Fig. 8. The arrow shows that it is only a lower limit due to the large uncertainty in contamination which affects in particular the PMS phases. The number of stars redder than $(U-V)>0$ in the observed CMD is much larger than in all the simulations which reproduce correctly the number of stars in the blue plume. Since this ratio is low, even after the foreground contamination subtraction, it points, independently from the evidence on number of MS stars, toward long $\tau_{\max }$.

\subsection{The initial mass function}

The observations of the young stars on the MS can be used to constrain the initial mass function for the range of masses observed, $M \gtrsim 5 M_{\odot}$. The comparison of the simulated and observed luminosity functions along the MS is presented for a set of models with on-going SF lasting for 100, 50, 30, and $10 \mathrm{Myr}$, as well as for two models in which the SF was stopped 5 and $2.5 \mathrm{Myr}$ ago. Figure 9 compares the observed luminosity function (dots) with the models for $Z=0.008$ and four different IMF slopes: Salpeter IMF (slope $\alpha=2.35$; solid black line), Scalo IMF (slope $\alpha=2.6$ for $M \leq 3.5 M_{\odot}$; Scalo 1986; green short dashed line), IMF with slope $\alpha=2$ (blue long dashed line), and IMF with slope $\alpha=3$ (red dotted line).

The observed slope of the MS between $22.5<V<24.5$ is $\Delta \log N / \Delta V=0.47$ as derived by least square fitting of the 

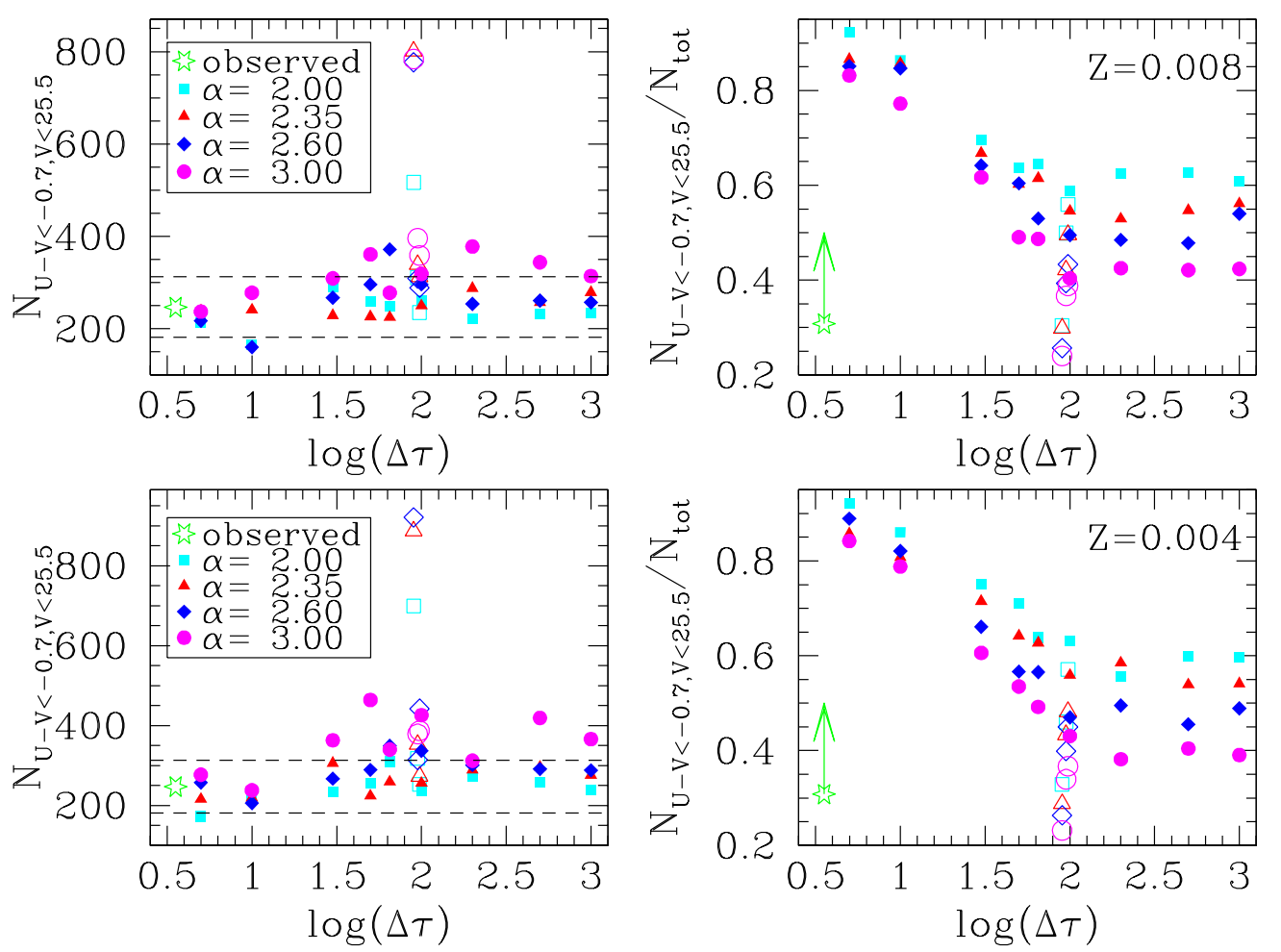

Fig. 8. Left panels: number of stars in the blue plume $(U-V<-0.7)$ for $V$ brighter than 25.5 vs. logarithm of star formation duration is shown for the models with $Z=0.008$ (top) and $Z=0.004$ (bottom). The observed number of stars within the same limits is indicated with a green star. Dotted lines indicate $3 \sigma$ Poissonian fluctuation limits in the number of observed and simulated stars. The $x$-axis value for the observed point is arbitrary. Different symbols are used for models with different IMF slopes as indicated in the legends. The models for which star formation was terminated 2.5, 5 and $10 \mathrm{Myr}$ ago are plotted with open symbols, and the models for which the star formation is still on-going have filled symbols. Right panels: the ratio of the blue plume vs. total number of simulated stars. The observed point (corrected for the foreground contamination) is only a lower limit due to a large uncertainty in foreground contamination.

observed luminosity function in this magnitude range. The results for the models with $Z=0.004$ and $Z=0.008$ are equivalent and we plot only $Z=0.008$ models in Fig. 9. The models with IMF slope $\alpha=2.35$ provide the best fit of the luminosity function for both metallicities, $Z=0.004$ and 0.008 . Slightly shallower $(\alpha=2)$ and steeper $(\alpha=2.6)$ slopes for IMF are also acceptable, while the $\alpha=3$ slope produces too many stars in the lower mass bins in all the simulations. Note that the models where the SF has stopped 2.5 Myr ago are well fitted with shallower IMFs, and they would exclude Scalo or steeper IMF slopes.

These results are confirmed by the $\chi^{2}$ tests applied to the luminosity functions of the blue stars and are also summarized in Fig. 8. In the left panels the dashed lines exclude the models with IMF slope $\alpha=3$, because the simulated number of blue plume stars is always larger than observed (having fixed in the simulations the number of stars in a box on the MS). In the right panels the above mentioned saturation value (or the inflexion point) of the number of PMS vs. the total number of stars depends on the IMF slope. The steeper the IMF, the more favoured the PMS. Even though the observed ratio would favour $\alpha=3.00$, this IMF slope is excluded by the excess number of stars in the blue plume, which is the more robust constraint. Therefore it seems that the contamination of the red part of the CMD is indeed much larger than that given by the Besançon counts as already noted by Rejkuba et al. (2001).
Additionally, there may be few compact background galaxies contaminating the red part of the CMD.

Our simulations do not include binary stars. The presence of binaries would increase the IMF slope by an amount that depends on the slope and the fraction of stellar mass in binary or multiple stellar systems. According to Sagar \& Richtler (1991) if the fraction of binaries is more than $50 \%$ the IMF slope increases by 0.3 to 0.4 . As the binary fraction in our field is not known, it is possible that the actual IMF slope is somewhat steeper than $\alpha=2.35$.

\section{Young stellar population in the VK CMD}

Figure 10 shows $V K$ optical-near IR CMD for this field constructed from the VLT FORS $1 V$-band and ISAAC $K$ s-band photometry published by Rejkuba et al. (2001). Most of the stars are distributed along the red giant branch and are thus older than $\sim 1$ Gyr. There is also a large number of bright AGB stars, including several hundred Mira variables, located above the tip of the RGB (Rejkuba et al. 2003). Some $\sim 110$ stars with colors $2 \lessgtr V-K \lesssim 4$ are expected to belong to foreground Galactic population. Several dozens of stars in the bluest part of the diagram $(V-K<2)$ do not appear in the control halo field (see Rejkuba et al. 2001). They have very small photometric errors $(90 \%$ has $\sigma<0.2)$, and are bright in the optical. All but two stars are detected in the $U$-band images as 


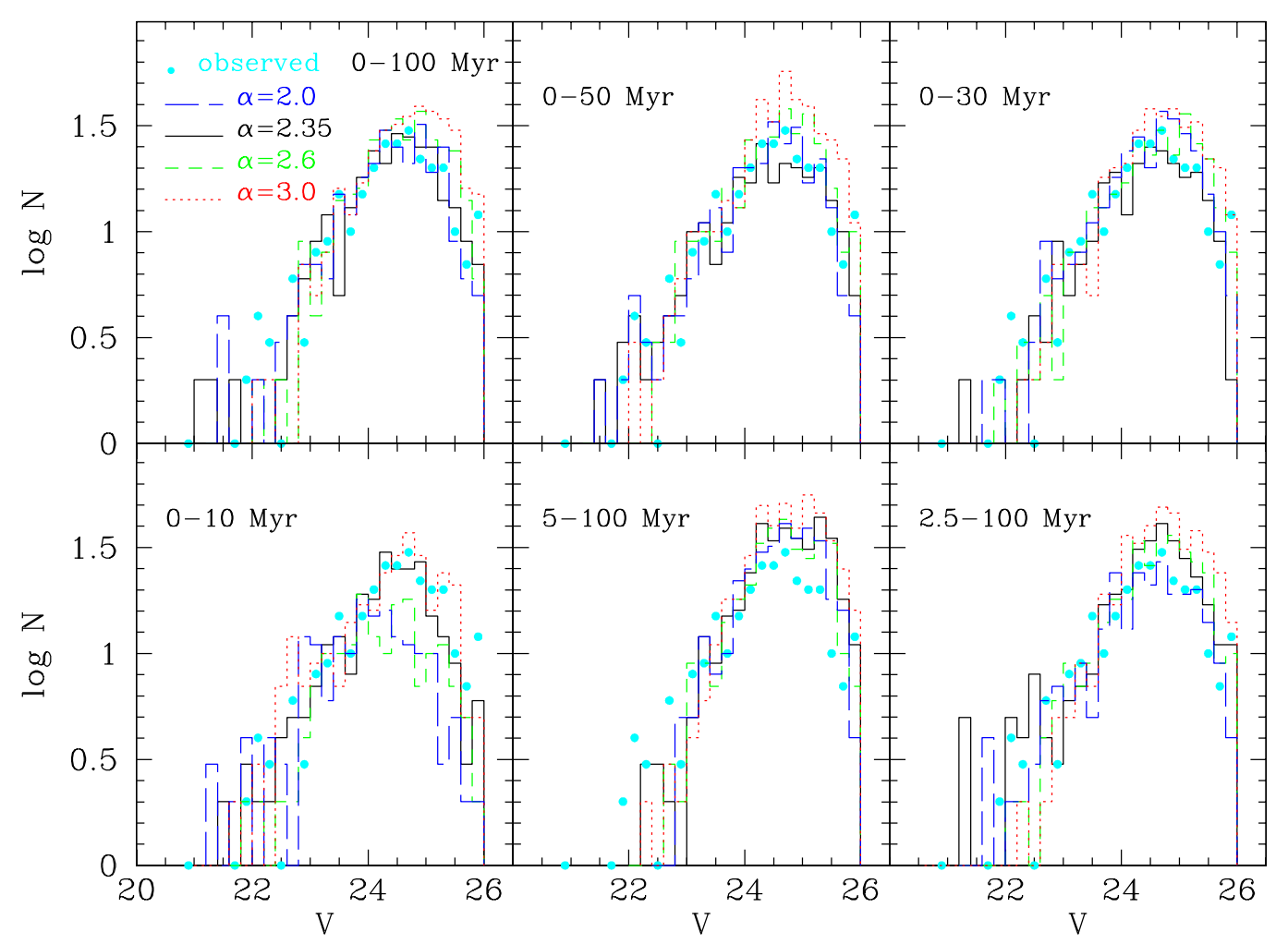

Fig. 9. The observed MS luminosity function (cyan dots) is compared with synthetic luminosity functions for different $\mathrm{SFHs}$ for $Z=0.008$ and four different IMF slopes $(\alpha=2.0,2.35,2.6$, and 3.0) as indicated in the legend. Synthetic luminosity functions correspond to models calculated adopting the appropriate distance, reddening and a constant SFR in the interval that is indicated in each panel (beginning-end of the burst). In the observed and simulated luminosity functions color and magnitude cuts are applied, and only stars bluer than $U-V=-0.7$ and brighter than $V=26$ are compared.
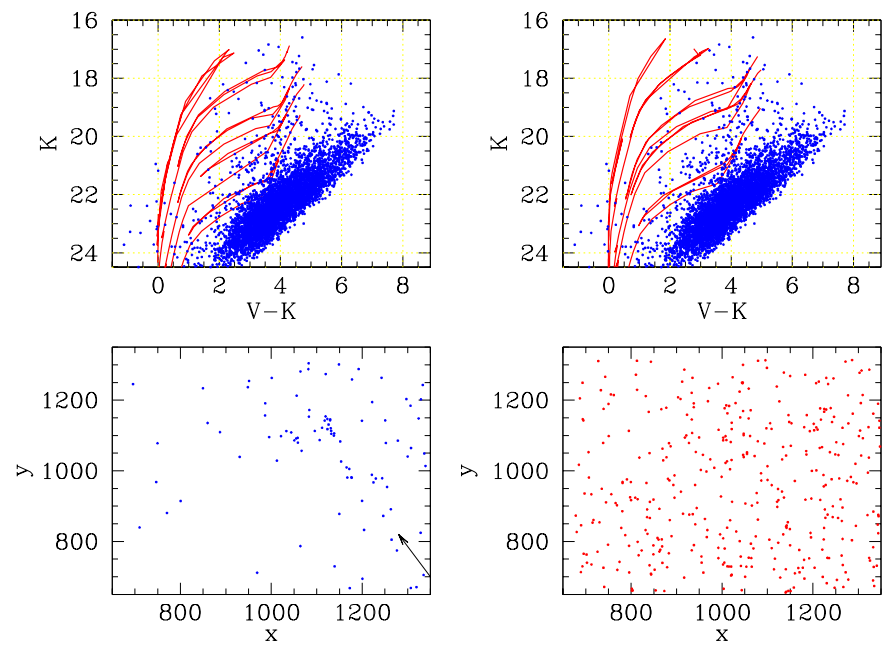

Fig. 10. The comparison of the the $V K$ CMD with stellar evolutionary tracks for $Z=0.004$ (upper left) and $Z=0.008$ (upper right panel) and masses of $6,9,12,20$ and $40 M_{\odot}$. In the lower panels the spatial distribution of the blue $(V-K<2$ and $K<24)$ stars on the left and red $(V-K>5$ and $19<K<21)$ stars on the right is shown. Note the alignment of the blue stars along the direction of the radio jet indicated by the arrow in the lower right corner of the left panel.

well. We plot the spatial distribution of the blue $(V-K<2$ and $K<24)$ and red $(V-K>5$ and $19<K<21)$ stars in the shell field in Fig. 10. While the reddest objects spread evenly over the field, the majority of the blue stars are aligned in the direction of the jet just like in the optical CMDs.

The low number of young stars observed in the much smaller field of view of ISAAC prevents us from drawing conclusions from their observations. The comparison with the Padova stellar evolutionary tracks (in the upper panels of Fig. 10) for two different metallicities $Z=0.004$ (left) and $Z=0.008$ (right), indicates again a very small difference between the models.

The $V K$ CMD gives complementary information about the older stellar population in the halo. However, due to large incompleteness in the red parts of this CMD (see Rejkuba et al. 2001), the SFH of the old stars in NGC 5128 will be investigated with deeper HST+ACS observations.

\section{Discussion and conclusions}

The recent star formation history in the north-eastern halo field in the giant elliptical galaxy NGC 5128 was investigated with the means of synthetic CMDs. The comparison of the isochrones and the data has to be done with great care, using the correct filter transmission curves to compute a consistent set of bolometric corrections. The $U$-band is known to be the most difficult to reproduce due to the presence of the atmospheric cut-off, to the presence of the Balmer break within that band, and to strong blanketing by metals. Additionally due to large differences between the $U$-band filters and a possible 
miss-match in stellar properties $\left(\log g, T_{\text {eff }}\right.$ and $\left.[\mathrm{M} / \mathrm{H}]\right)$ between the calibrators and program objects, it is possible to introduce systematic errors if the calibrators do not rely on the same photometric system as the observations. This affects mostly our reddening estimate and very little other results.

The new reddening estimated by matching the locus of the observed MS in the $U-V$ plane with the models, using the corrected bolometric corrections for $U$ passband and assuming the Galactic extinction law (Cardelli et al. 1989) is $E(B-V)=0.35$, significantly larger than previously obtained $E(B-V)=0.15$ (Rejkuba et al. 2001). It should be noted, though, that Rejkuba et al. estimate is obtained from the comparison of mostly foreground stars with isochrones, and is in fact in good agreement with $E(B-V)=0.11$ from Schlegel et al. (1998) maps. This implies a rather significant internal extinction in this field. While some internal extinction in the areas of recent star formation could be expected, we would like to stress that this result has large uncertainty due to above mentioned difficulties to reproduce correctly the $U$-band. The maximum possible systematic error introduced by the calibration to the Landolt photometric system is of the order of $0.04-0.08$ in the $U$-band for the bluest stars. This translates to a possible over-estimate of reddening of $E(B-V)=0.07-0.14$. Assuming the maximum possible systematic error, the internal extinction in this field is reduced to about $E(B-V) \sim 0.10$.

Unfortunately, on the basis of the $U$ - and $V$-band photometry alone it is not possible to constrain the metallicity of the stars due to reddening-distance-metallicity degeneracy of the models. However, the comparison of the synthetic and observed CMDs gave a number of quantitative constraints to the recent $\mathrm{SFH}$ in the studied halo field.

We have constructed simulated CMDs of young stars in the halo, having fixed the number of stars in a box on the bright portion of the MS, for two metallicities $Z=0.004$ and $Z=$ 0.008 . The parameters that were varied were $\tau_{\max }$ (the starting epoch of the SF), $\tau_{\min }$ (the ending epoch of the SF), and $\alpha$ (the slope of the IMF).

The blue MS stars and their luminosity function constrain $\tau_{\text {min }}$ to be smaller than $\sim 2.5 \mathrm{Myr}$ and exclude the IMF slopes steeper than $\alpha \gtrsim 2.6$. The ratio of the blue vs. total number of stars favours steeper IMF slope and longer star formation duration ( $\tau_{\max } \gtrsim 100 \mathrm{Myr}$ ). This is a robust result and is not affected by the systematic errors in calibration, as the slope of the MS does not change. The exact ratio of the blue vs. total number of stars may be affected by the systematics, but not so much to change our conclusions.

From the simulations with $\tau_{\min }=0$ and $\tau_{\max }=100 \mathrm{Myr}$, the total extracted mass in stars in the range of masses $0.6 \leq$ $M \leq 100 M_{\odot}$ is $\sim 0.4,0.7$, or $1.3 \times 10^{6} M_{\odot}$, for IMF slopes of 2 and 2.35 , or 2.6, respectively. The SFR associated with these IMFs and mass limits are approximately 0.004, 0.007, and $0.013 M_{\odot} \mathrm{yr}^{-1}$, for the three IMF slopes in the above order, and it is independent of $\tau_{\max }$. Moreover the smooth appearance of the MS, the absence of gaps and breaks in the slope of the MS luminosity function, indicates that most probably no major fluctuations in the SFR have occurred over the sampled time interval. The reported values of the SFR depend on a combination of the MS and PMS stars, for which the possible systematic errors in magnitudes (that go in different direction for these two groups) partially cancel out. We estimate that our results are not changed strongly by the systematics in calibration.

At the distance of $3.6 \mathrm{Mpc}$, the observed field surveys a projected area of approximately $45 \mathrm{kpc}^{2}$, so that the derived star formation rates range from $\sim 9 \times 10^{-5}$ to $\sim 3 \times$ $10^{-4} M_{\odot} \mathrm{yr}^{-1} \mathrm{kpc}^{-2}$. This is lower than the typical SFR quoted for the solar neighbourhood $\left(4.2 \times 10^{-3} M_{\odot} \mathrm{yr}^{-1} \mathrm{kpc}^{-2}\right.$; Timmes et al. 1995), and is on the low side of the SFRs derived via theoretical simulations for dwarf irregular galaxies in the Local Group (ranging from approximately $10^{-4}$ to $10^{-2} M_{\odot} \mathrm{yr}^{-1} \mathrm{kpc}^{-2}$; Marconi et al. 1995; Tolstoy 1996; Gallart et al. 1996a). For example, the star formation rate in WLM dIrr within the last million years was $1.3 \times 10^{-3} M_{\odot} \mathrm{yr}^{-1} \mathrm{kpc}^{-2}$ (Dolphin 2000).

On a more speculative side, it is possible to estimate the maximum duration of the future star formation. The fuel for this recent star formation is provided by the large HI gas cloud in the vicinity of the field (Schiminovich et al. 1994). Assuming the constant $S F R$ and a $100 \%$ efficiency in converting gas to stars, we can estimate the gas depletion timescale as:

$\tau_{\mathrm{g}}=\frac{M_{\mathrm{HI}}}{S F R}$

In the north-eastern shell very close to where the star formation is observed, $2.1 \times 10^{7} M_{\odot}$ of HI have been detected (Schiminovich et al. 1994) as well as $1.7 \times 10^{7} M_{\odot}$ of $\mathrm{H}_{2}$ (Charmandaris et al. 2000). At the constant star formation level of $0.006 M_{\odot} \mathrm{yr}^{-1}$ it would take $\sim 100 \mathrm{Myr}$ to use up all the molecular gas and $\sim 230 \mathrm{Myr}$ to use all the hydrogen.

Our results place a lower limit of $\sim 100 \mathrm{Myr}$ to the last merging event and a lower limit to the time of the formation of the jet in Cen A. To set better constraints on the starting epoch of the last SF episode deeper CMDs and a more robust determination of the foreground contamination are necessary.

Acknowledgements. We thank D. Minniti and D. Silva for useful comments. We acknowledge an anonymous referee for his/her very useful comments, and especially for pointing out the uncertainty of the photometry due to the calibration procedure.

\section{Appendix A: Random extraction of a star with a given mass and age}

We use a Monte Carlo algorithm to randomly select a mass and an age according to a given distribution. The mass is extracted according to a single slope (e.g. Salpeter IMF; Salpeter 1955),

$\mathrm{d} N=A M^{-\alpha} \mathrm{d} M$

or double slope initial mass function (e.g. Gould IMF; Gould et al. 1997) with slope $\alpha_{1}$ within a mass range $M_{1} \leq M \leq M_{2}$ and $\alpha_{2}$ within $M_{2} \leq M \leq M_{3}$. Here " $A$ " is the normalisation factor that depends on the upper and lower mass limits used in the models. The choice of the slope is a free parameter.

The age distribution is adopted to be either flat, representing a constant star formation rate,

$\Psi(t)=C \quad$ for $\quad \tau_{\min } \leq t \leq \tau_{\max }$ 
or exponentially decreasing,

$\Psi(t)=C \mathrm{e}^{-t / \tau} \quad$ for $\quad \tau_{\min } \leq t \leq \tau_{\max }$

with given limits for the age of the oldest stars $\left(\tau_{\max }\right)$ and for the duration of the star formation episode $\left(\Delta \tau=\tau_{\max }-\tau_{\min }\right)$.

We have used a single metallicity simulator to produce synthetic CMDs for the young stellar population. This is a good approximation, because the spread in ages of the young stars (few $\times 10 \mathrm{Myr}$ ) is too short for a significant metal enrichment to occur. However in the simulation of the optical-near IR CMDs that are dominated by the old and intermediate-age population, evolution of the metallicity would have to be assumed.

\section{Appendix B: Interpolation of the stellar evolutionary models}

We have adopted two different sets of models: Padova models (Fagotto et al. 1994) and models computed by Cassisi \& Salaris (1997) and extended by Bono et al. (1997a, 1997b). For the simulations of single metallicity star formation histories used to compare with the youngest stars in NGC 5128, we use the Padova models by Fagotto et al. (1994). These models have tracks for stellar masses ranging from 0.6 to $120 M_{\odot}$. The newer set of Padova models (Girardi et al. 2000) are not computed for stars with masses larger than $7 M_{\odot}$ such as those that are observed in the north-eastern halo field of NGC 5128.

Given the extracted mass and age, the luminosity and temperature of the star were determined via the linear interpolation in a set of evolutionary tracks. Each track consists of a number of points (age, mass, $\left.\log \left(L / L_{\odot}\right), \log \left(T_{\text {eff }}\right)\right)$. We have divided each track into portions according to stellar evolutionary phase, and interpolated linearly between the adjacent tracks within the same portion. Typically, the portions of the tracks are:

- Main sequence (MS) - ending either at core H exhaustion or at the start of the first runaway expansion;

- Subgiant branch - ending at the base of the RGB;

- RGB - ending at the tip of the RGB;

- Horizontal branch - ending either at the core He exhaustion or at the base of the AGB;

- Early AGB - ending at the first thermal pulse.

For the high mass stars $\left(M \geq 20 M_{\odot}\right)$, whose evolution is affected by mass loss, we divide the evolution in two major phases (MS and post main sequence), and choose conveniently the description of the post main sequence (PMS) phase, inserting further sub-phases when needed.

In most of the cases the $\log (L)$ and $\log \left(T_{\text {eff }}\right)$ of the extracted star are interpolated between the values of adjacent masses read at the same fractional age in the appropriate evolutionary phase:

$t_{p h}=\left(t-t_{0}\right) /\left(t_{1}-t_{0}\right)$

where $t_{0}$ and $t_{1}$ are respectively the ages at the beginning and at the end of the appropriate evolutionary phase and $t$ is the age of the extracted object. In few evolutionary phases when luminosity and/or temperature presented a maximum or minimum at variable $t_{p h}$, we inserted a break $t_{p h}\left(t_{p h, \mathrm{br}}\right)$ and effectively divided that phase into two sub-phases. $\log (L)$ and $\log \left(T_{\text {eff }}\right)$ of the extracted star at the extracted age are then found by interpolation at:

$t_{p h} / t_{p h, \mathrm{br}}$ for ages $t_{p h} \leq t_{p h, \mathrm{br}}$

$\left(t_{p h}-t_{p h, \mathrm{br}}\right) /\left(1-t_{p h, \mathrm{br}}\right)$ for ages $t_{p h} \geq t_{p h, \mathrm{br}}$.

When the interpolation has to be done also in metallicity, the simple linear interpolation algorithm described above is not enough. It is necessary to perform a bilinear interpolation, because the stars of the same masses, but different metallicities have different ages. Thus for each extracted mass one has to first interpolate in $\log (L)$ and $\log \left(T_{\text {eff }}\right)$ for a given age, and then again for a given metallicity. In order to simplify the problem Zoccali et al. (2003) adopted a set of isochrones as a starting point. Using the tabulated values of $\log (L)$ and $\log \left(T_{\text {eff }}\right)$ for stars of given mass in an isochrone the interpolation is done linearly within isochrones of the same age and different metallicities. The Padova models are not adequate for these simulations due to the very low number of points in each isochrone (approx. 30-55 points for each track) and it is better to use the Cassisi models (Cassisi \& Salaris 1997; Bono et al. 1997a, 1997b) which have much more detailed evolution with more than 600 points per track. Given a mass and metallicity, the luminosity and temperature of the star are determined through the linear interpolation in a set of isochrones. The interpolation in $\log (L)$ and $\log \left(T_{\text {eff }}\right)$ is made for the mass that has the same evolutionary phase of the extracted mass:

$p h=m / m_{1} \quad$ for objects on the main sequence

$p h=m_{0}+\left(m-m_{0}\right) /\left(m_{1}-m_{0}\right) \quad$ for later phases

where $m_{0}$ and $m_{1}$ are the masses at the beginning and the end of the evolutionary phase. The evolutionary phases are as before: main sequence, subgiant branch, RGB, core-helium burning and AGB.

\section{References}

Bertelli, G., Mateo, M., Chiosi, C., \& Bressan, A. 1992, ApJ, 388, 400 Bertelli, G., Bressan, A., Chiosi, C., Fagotto, F., \& Nasi, E. 1994, A\&AS, 106, 275

Bessell, M. S., PASP, 107, 672

Bessell, M. S., Castelli, F., \& Plez, B. 1998, A\&A, 333, 231

Bono, G., Caputo, F., Cassisi, S., Castellani, V., \& Marconi, M. 1997, ApJ, 489, 822

Bono, G., Caputo, F., Cassisi, S., Castellani, V., \& Marconi, M. 1997, ApJ, 479, 279

Buser, R., \& Kurucz, R. L. 1978, A\&A, 70, 555

Cardelli, J. A., Clayton, G. C., \& Mathis, J. S. 1989, ApJ, 345, 245

Cassisi, S., \& Salaris, M. 1997, MNRAS, 285, 593

Castelli, F., Gratton, R. G., \& Kurucz, R. L. 1997, A\&A, 318, 841

Charmandaris, V., Combes, F., \& van der Hulst, J. M. 2000, A\&A, 356, L1

Cole, A. A., Tolstoy, E., Gallagher III, J. S., et al. 1999, AJ, 118, 1657

Cuby, J. G., Lidman, C., Johnson, R., \& Moutou, C. 2003, ISAAC User Manual

Davidge, T. J., \& van den Bergh, S. 2001, ApJ, 553, L133

Dolphin, A. 1997, New Astron., 2(5), 397 
Dolphin, A. E. 2000, ApJ, 531, 804

Fagotto, F., Bressan, A., Bertelli, G., \& Chiosi, C. 1994, A\&AS, 105, 29

Fassett, C. I., \& Graham, J. A. 2000, ApJ, 538, 594

Ferraro, F. R., Fusi Pecci, F., Tosi, M., \& Buonanno, R. 1989, MNRAS, 241, 433

Gallart, C., Aparicio, A., Bertelli, G., \& Chiosi, C. 1996a, AJ, 112, 1950

Gallart, C., Aparicio, A., Bertelli, G., \& Chiosi, C. 1996b, AJ, 112, 2596

Girardi, L., Bressan, A., Bertelli, G., \& Chiosi, C. 2000, A\&AS, 141, 1

Girardi, L., Bertelli, G., Bressan, A., et al. 2002, A\&A, 391, 195

Gould, A., Bahcall, J. N., \& Flynn, C. 1997, ApJ, 482, 913

Graham, J. A. 1998, ApJ, 502, 245

Graham, J. A., \& Price, R. M., ApJ, 247, 813

Greggio, L., Tosi, M., Clampin, M., et al. 1998, ApJ, 504, 725

Harris, G. L. H., Harris, W. E., \& Poole, G. B. 1999, AJ, 117, 855

Harris, J., \& Zaritsky, D. 2001, ApJS, 136, 25

Hernández, X., Valls-Gabaud, D., \& Gilmore, G. 1998, MNRAS, 304, 705

Hurley-Keller, D., Mateo, M., \& Nemec, J. 1998, AJ, 115, 1840

Israel, F. P. 1998, A\&AR, 8, 237

Johnson, H. L., \& Morgan, W. W. 1953, ApJ, 117, 313

Kurucz, R. L. 1993, IAU Symp. 149, The Stellar Populations of Galaxies, ed. B. Barbuy, \& A. Renzini (Dordrecht: Kluwer), 225 Landolt, A. U. 1992, AJ, 104, 340

Marconi, G., Tosi, M., Greggio, L., \& Focardi, P. 1995, AJ, 109, 173

Montegriffo, P., Ferraro, F. R., Origlia, L., \& Fusi Pecci, F. 1998, MNRAS, 297, 872

Morganti, R., Killeen, N. E. B., Ekers, R. D., \& Oosterloo, T. A. 1999, MNRAS, 307, 750

Morossi, C., Franchini, M., Malagnini, M. L., Kurucz, R. L., \& Buser, R. 1993, A\&A, 277, 173
Mould, J. R., Ridgewell, A., \& Gallagher, J. S., III, et al. 2000, ApJ, 536, 266

Olsen, K. A. G. 1999, AJ, 117, 2244

Partridge, R. B., \& Peebles, P. J. E. 1967, ApJ, 147, 868

Rejkuba, M. 2004, A\&A, 413, 903

Rejkuba, M., Minniti, D., Bedding, T., \& Silva, D. R. 2001, A\&A, 379, 781

Rejkuba, M., Minniti, D., Courbin, F., \& Silva, D. R. 2002, ApJ, 564, 688

Rejkuba, M., Minniti, D., \& Silva, D. R. 2003, A\&A, 406, 75

Renzini, A., Greggio, L., Ritossa, C., \& Ferrario, L. 1992, ApJ, 400, 280

Ritossa, C. 1996, MNRAS, 281, 970

Robin, A., \& Creze, M. 1986, A\&A, 157, 71

Robin, A. C., Haywood, M., Creze, M., Ojha, D. K., \& Bienayme, O. 1996, A\&A, 305, 125

Sagar, R., \& Richtler, T. 1991, A\&A, 250, 324

Salpeter, E. E. 1955, ApJ, 121, 161

Scalo, J. M. 1986, Fundamentals of Cosmic Physics, 11, 1

Schiminovich, D., van Gorkom, J. H., van der Hulst, J. M., \& Kasow, S. 1994, ApJ, 423, L101

Schlegel, D. J., Finkbeiner, D. P., \& Davis, M. 1998, ApJ, 500, 525

Schulte-Ladbeck, R. E., Hopp, U., Greggio, L., \& Crone, M. M. 2000, AJ, 120, 1713

Schulte-Ladbeck, R. E., Drozdovsky, I. O., Belfort, M., \& Hopp, U. 2003, Ap\&SS, 284, 909

Soria, R., Mould, J. R., Watson, A. M., et al. 1996, ApJ, 465, 79

Szeifert, T. 2002, FORS1+2 User Manual

Timmes, F. X., Woosley, S. E., \& Weaver, T. A. 1995, ApJS, 98, 617

Tolstoy, E. 1996, ApJ, 462, 684

Toomre, A. 1977, The Evolution of Galaxies and Stellar Populations, ed. B. Tinsley \& R. Larson (New Haven: Yale Univ. Press), 401

Tosi, M., Greggio, L., Marconi, G., \& Focardi, P. 1991, AJ, 102, 951

Zoccali, M., Renzini, A., Ortolani, S., et al. 2003, A\&A, 399, 931 